\section{Pesquisa sobre as Condições de Saúde Bucal da População Brasileira (SB Brasil 2003): seus dados não produzem estimativas populacionais, mas há possibilidade de correção}

\author{
Brazilian Oral Health Survey (SB Brazil 2003): \\ data do not allow for population estimates, \\ but correction is possible
}

\begin{abstract}
The Brazilian Oral Health Survey (SB Brazil 2003) was the most comprehensive study on oral health conditions ever conducted in Brazil. Probabilistic sampling methods were applied in order for the collected data to represent the population age groups selected in the 5 regions of the country. However, this was not possible because the sampling process was never concluded, which would require estimation of the sample weights and identification of the sample's other structural variables (selection strata and primary sampling units). This paper describes the SB Brazil 2003 sample design, formulates the inclusion probabilities in the multiple selection stages, and proposes strategies for estimating the sample weights. The strategy to define the sample weights and identify the sample's structural variables hinges on retrieving data that should have been recorded in the reports produced during the survey, and in their absence, in information available from the Brazilian Institute of Geography and Statistics (IBGE) and Ministry of Education and Culture (MEC), as valid proxies.
\end{abstract}

Oral Health; Sampling Studies; Health Status; Population
Rejane Christine de Sousa Queiroz 1

Margareth Crisóstomo Portela 1

Mauricio Teixeira Leite de Vasconcellos 2

\section{Introdução}

O processo de inserção da área de saúde bucal na agenda da saúde pública brasileira trouxe a necessidade de ampliar o conhecimento das condições de saúde bucal da população brasileira e de quantificar os eventos de interesse para apoiar o planejamento de ações na área. A partir da década de 80 do século passado, a área começou a acumular experiência em grandes inquéritos epidemiológicos que incluíam exames clínicos bucais dos entrevistados, especialmente escolares.

O primeiro inquérito sobre a saúde bucal no Brasil foi realizado em $1986{ }^{1}$ pela Divisão Nacional de Saúde Bucal (DNSB) do Ministério da Saúde, com o apoio do Instituto de Planejamento Econômico e Social da Secretaria de Planejamento da Presidência da República. As limitações orçamentárias foram determinantes para definir a abrangência geográfica, a população alvo e a abrangência do exame bucal. Realizou-se um inquérito na área urbana de 16 capitais, distribuídas entre as cinco macrorregiões do país, incluindo crianças (6 a 12 anos), adolescentes (15 a 19 anos) e adultos (35 a 44 anos e 50 a 59 anos). A justificativa para não considerar idosos entre $65 \mathrm{e}$ 74, como proposto pela Organização Mundial da Saúde (OMS) e adotado em países desenvolvidos (65 a 74 anos), foi baseada no fato de a esperança de vida ao nascer ser, à época, de 65,5 anos no Brasil 2. Ocorrência de cárie e doença periodon- 
tal, necessidade e uso de prótese dentária foram observados, além de informações sobre acesso aos serviços de saúde.

Nesse inquérito, a idade de 12 anos foi utilizada para fins de comparação internacional, obedecendo a critérios propostos pela Federação Dentária Internacional (FDI) e pela OMS, que também lançaram metas com índices a serem alcançados por volta do ano de 20003.

O relatório desse inquérito aponta várias dificuldades enfrentadas para a sua efetivação, incluindo questões operacionais (dimensão e a diversidade regional do país) e a necessidade de obter dados mais acurados para o planejamento governamental, diante da magnitude da necessidade de tratamento odontológico ${ }^{2}$ pela população.

Em relação ao procedimento de amostragem, o relatório informa que foram utilizadas duas amostras, uma para a faixa etária entre 6 e 12 anos e outra para as demais faixas etárias. As amostras foram selecionadas em dois estágios. Para estudantes (6 a 12 anos) foram selecionados estabelecimentos de ensino (1o e 2o graus) e, neles, os estudantes que foram entrevistados e examinados. Para as demais faixas etárias, foram selecionados setores censitários; neles, os domicílios onde foram entrevistados e examinados todos os moradores das demais faixas etárias contempladas.

Embora restrito à área urbana de algumas capitais, este primeiro inquérito representou um passo importante, na medida em que somou conhecimentos específicos das áreas de Odontologia e Saúde Pública aos métodos e técnicas do Instituto de Pesquisa Econômica Aplicada (IPEA) e do Instituto Brasileiro de Geografia e Estatística (IBGE).

Dez anos depois, em 1996, com o Sistema Único de Saúde (SUS) já implantado, foi realizado o segundo inquérito sob coordenação da Área Técnica de Saúde Bucal do Ministério da Saúde. O seu objetivo foi verificar as alterações ocorridas no perfil de saúde bucal da população brasileira, tomando como referência o inquérito de 1986, e obter um referencial para as ações preventivas do SUS 4 . Apesar de ampliar a abrangência geográfica (27 capitais das Unidades da Federação), o inquérito limitou-se ao exame de cárie na faixa etária de 6 a 12 anos, com crianças selecionadas em escolas públicas e privadas 5 . Ainda que houvesse dados disponíveis no Departamento de Informática do SUS (DATASUS; http:/ / tabnet. datasus.gov.br/cgi/sbucal/sbdescr.htm, acessado em 12/Dez/2007), a pesquisa sequer gerou um relatório final.

Embora obtivessem dados em diferentes regiões do país, os dois inquéritos não foram sufi- cientes para a construção de um quadro abrangente sobre as condições de saúde bucal da população brasileira. Eles representavam as características dos subgrupos populacionais definidos pelas faixas etárias consideradas e residentes nas cidades pesquisadas; não eram representativos da população das regiões e, muito menos, da população brasileira.

O reconhecimento dessas limitações conduziu o Ministério da Saúde a conceber um projeto para enfrentar o desafio de realizar um diagnóstico de saúde bucal do país, contemplando um número maior de eventos e de grupos etários. Nasce, então, o projeto SB Brasil 2003, com abrangência realmente nacional, incorporando, pela primeira vez, as faixas etárias de 18 a 36 meses e 65 a 74 anos, e a área rural. Apesar do título, esse terceiro inquérito foi realizado entre 2002 e 2003, tendo sido concebido para obter informações epidemiológicas capazes de subsidiar a elaboração das diretrizes para uma política nacional de saúde bucal, bem como ações para o fortalecimento da gestão dos serviços públicos de saúde bucal nas diferentes esferas de governo.

Comparado aos inquéritos anteriores, o SB Brasil 2003 incluiu ainda uma gama maior de dados relativos à situação sócio-econômica, auto-avaliação da saúde bucal e acesso aos serviços odontológicos. Além disso, foram realizados exames clínicos que, em princípio, permitiriam comparar a situação observada com as metas de saúde bucal preconizadas para o ano 2000 pela OMS 6.

O planejamento deste inquérito considerou a seleção de uma amostra probabilística por conglomerados, representativa de faixas etárias da população das cinco macrorregiões. O processo de amostragem, entretanto, não foi concluído. Faltou o cálculo dos pesos amostrais necessários para a produção de estimativas válidas para a população brasileira.

Em 2004, houve a publicação dos seus principais resultados pelo Ministério da Saúde 6. A disseminação do banco de dados associado à descrição de que a pesquisa "permitiu a produção de inferências para cada uma das macrorregiões brasileiras, por porte do município e para cada idade ou grupo etário" 6 (p. 13) talvez tenha induzido o uso dos dados do SB Brasil 2003 por diversos pesquisadores, que publicaram artigos científicos em revistas nacionais e internacionais $7,8,9,10$, sem perceberem que os resultados não eram inferências estatisticamente válidas para os grupos etários da população de pesquisa (brasileira ou por macrorregião) porque eram referidos e limitados ao grupo estudado (estatísticas da amostra). 
Este artigo tem por objetivo demonstrar que o procedimento de amostragem do SB Brasil 2003 não foi concluído, alertando seus potenciais usuários de que estimativas produzidas não são representativas da população brasileira, além de apontar para as possibilidades de correção dos problemas identificados. O artigo não avança na correção dos problemas indicados, pois ela depende do resgate de informações constantes dos relatórios das equipes de campo, o que necessariamente passa pelo apoio do Ministério da Saúde e das coordenações estaduais e municipais do projeto SB Brasil 2003.

Por que as estimativas do SB Brasil 2003 não são válidas para a população?

Desde que Kiar 11 apresentou suas idéias sobre o método representativo, hoje conhecido como amostragem probabilística, pesos amostrais são utilizados para a produção de inferências sobre a população de pesquisa ou população amostrada, como definiu Cochran 12 . Na amostragem probabilística cada unidade da amostra tem uma probabilidade de inclusão na amostra, representada por $\pi_{\mathrm{i}}$, cujo inverso é o peso amostral $\left(\mathrm{w}_{\mathrm{i}}\right)$, ou seja, $\mathrm{w}_{\mathrm{i}}=\pi_{\mathrm{i}}^{-1}$.

Como a base de dados do SB Brasil 2003 não tem pesos amostrais, os resultados desta pesquisa são representativos do grupo de 108.921 pessoas examinadas, não podendo ser extrapolados para a população de pesquisa (população brasileira dos grupos etários estudados).

É fato que alguns desenhos de amostra são concebidos de forma que as probabilidades de seleção sejam constantes para todas as unidades da amostra $\left(\pi_{\mathrm{i}}=\pi \forall \mathrm{i} \in \mathrm{S}\right.$, onde $\mathrm{S}$ é a amostra). Esses planos, ditos autoponderados 12, têm como propriedade principal o fato de médias, razões, proporções e prevalências amostrais serem estimativas não enviesadas da população, o que não vale para totais. No entanto, este não é o caso da amostra do SB Brasil 2003, cujo desenho forçosamente conduz a pesos desiguais.

Na Tabela 1, são apresentados pesos relativos que, apesar de grosseiros, dão uma idéia de quantas pessoas da população cada pessoa entrevistada representaria se o SB Brasil 2003 tivesse sido realizado em 2000 com um esquema de seleção equiprovável em cada estrato da amostra. Os pesos relativos, calculados dividindo a população observada no Censo Demográfico de 2000 (IBGE; http://www.ibge.gov.br) pelo tamanho da amostra em cada estrato, variam entre 17,8 (12 anos, Centro-Oeste em municípios com população entre 50.001 a 100.000 habitantes) e
16.137,5 (35 a 44 anos, em municípios de mais de 100.000 habitantes do Sudeste).

Para cada grupo etário, a variação dos pesos relativos é bem grande, conforme indica a Tabela 2. Mesmo para estimativas de um único grupo etário e uma única macrorregião, a variação dos pesos relativos não é desprezível, fato que indica que as estatísticas da amostra constituem-se em estimativas enviesadas da população correspondente, com maior ou menor viés em função da variabilidade observada para cada variável.

É certo que para variáveis com reduzida variação na amostra as estatísticas amostrais, embora enviesadas, não serão muito diferentes das estimativas obtidas de forma correta; esse é possivelmente o caso da variável cárie dentária, de elevada prevalência na maioria dos grupos estudados. Contudo, quanto maior for a variabilidade da variável estudada, maior será o viés da estatística amostral em relação à estimativa correspondente. Além deste problema, há que se considerar que a falta dos pesos amostrais inviabiliza a estimação de totais populacionais.

Infelizmente, os problemas da amostra do SB Brasil 2003 não se limitam à ausência dos pesos amostrais. Para estimar variâncias e demais estatísticas dela dependentes (erros padrão, intervalos de confiança, significância de parâmetros de modelos estatísticos, dentre outras) é necessário, também, conhecer as outras informações estruturais do desenho da amostra: o estrato de seleção e a unidade primária de amostragem. Isto deriva do fato de o plano de amostragem do SB Brasil 2003 ser complexo 13, combinando estratificação, conglomeração e seleção com probabilidades desiguais. Ou seja, um plano amostral que não permite estimação baseada na inferência estatística clássica, visto não ser aplicável um de seus pressupostos básicos: o de que todas as unidades da amostra derivam de observações independentes e igualmente distribuídas.

Entretanto, partindo-se do pressuposto de que as instruções de coleta foram estritamente observadas por toda a equipe de campo, podese buscar informações exatas, previstas para constarem dos relatórios das equipes de campo, ou aproximações válidas derivadas de dados do IBGE e do Instituto Nacional de Estudos e Pesquisas Educacionais Anísio Teixeira (INEP) do Ministério da Educação (MEC), para determinar os pesos amostrais. 
Tamanho da amostra, população em 2000 e peso relativo por faixas etárias, segundo as macrorregiões e estratos de porte populacional.

\begin{tabular}{|c|c|c|c|c|c|c|c|c|c|}
\hline \multirow{3}{*}{$\begin{array}{l}\text { Macrorregiões e } \\
\text { estratos de porte } \\
\text { populacional } \\
\text { (habitantes) }\end{array}$} & \multicolumn{9}{|c|}{ Faixa etária } \\
\hline & & 18-36 meses & & & 5 anos & & & 12 anos & \\
\hline & Amostra & População * & $\begin{array}{c}\text { Peso } \\
\text { relativo ** }\end{array}$ & Amostra & População * & $\begin{array}{c}\text { Peso } \\
\text { relativo ** }\end{array}$ & Amostra & População * & $\begin{array}{c}\text { Peso } \\
\text { relativo ** }\end{array}$ \\
\hline Total & 12.117 & 6.474 .246 & 534,3 & 26.641 & 3.445 .580 & 129,3 & 34.550 & 3.524 .814 & 102,0 \\
\hline Norte & 3.305 & 651.066 & 197,0 & 4.678 & 335.414 & 71,7 & 6.208 & 314.984 & 50,7 \\
\hline Até 5.000 & 329 & 20.765 & 63,1 & 300 & 10.724 & 35,7 & 515 & 10.285 & 20,0 \\
\hline $5.001-10.000$ & 526 & 45.113 & 85,8 & 557 & 23.379 & 42,0 & 957 & 22.031 & 23,0 \\
\hline $10.001-50.000$ & 861 & 254.101 & 295,1 & 1.034 & 132.369 & 128,0 & 1.338 & 123.084 & 92,0 \\
\hline $50.001-100.000$ & 840 & 96.657 & 115,1 & 1.371 & 49.412 & 36,0 & 1.683 & 47.104 & 28,0 \\
\hline $100.000 \mathrm{ou}+$ & 749 & 234.430 & 313,0 & 1.416 & 119.530 & 84,4 & 1.715 & 112.480 & 65,6 \\
\hline Nordeste & 2.557 & 1.986 .462 & 776,9 & 4.580 & 1.073 .304 & 234,3 & 7.322 & 1.136 .394 & 155,2 \\
\hline Até 5.000 & 533 & 45.604 & 85,6 & 476 & 25.850 & 54,3 & 680 & 27.948 & 41,1 \\
\hline $5.001-10.000$ & 473 & 133.965 & 283,2 & 601 & 73.882 & 122,9 & 1.075 & 78.420 & 72,9 \\
\hline $10.001-50.000$ & 425 & 895.366 & $2.106,7$ & 660 & 491.400 & 744,5 & 1.451 & 516.364 & 355,9 \\
\hline $50.001-100.000$ & 634 & 268.025 & 422,8 & 1.443 & 144.236 & 100,0 & 2.114 & 151.974 & 71,9 \\
\hline $100.000 \mathrm{ou}+$ & 492 & 643.502 & $1.307,9$ & 1.400 & 337.936 & 241,4 & 2.002 & 361.688 & 180,7 \\
\hline Sudeste & 2.342 & 2.510 .795 & $1.072,1$ & 7.291 & 1.314 .709 & 180,3 & 8.052 & 1.357 .615 & 168,6 \\
\hline Até 5.000 & 447 & 55.179 & 123,4 & 457 & 30.882 & 67,6 & 604 & 33.195 & 55,0 \\
\hline $5.001-10.000$ & 545 & 105.913 & 194,3 & 586 & 58.906 & 100,5 & 1.030 & 62.855 & 61,0 \\
\hline $10.001-50.000$ & 660 & 472.142 & 715,4 & 1.334 & 257.414 & 193,0 & 1.871 & 271.194 & 144,9 \\
\hline $50.001-100.000$ & 255 & 253.497 & 994,1 & 2.445 & 135.976 & 55,6 & 2.216 & 142.885 & 64,5 \\
\hline $100.000 \mathrm{ou}+$ & 435 & 1.624 .064 & $3.733,5$ & 2.469 & 831.531 & 336,8 & 2.331 & 847.486 & 363,6 \\
\hline Sul & 2.725 & 873.180 & 320,4 & 6.042 & 480.144 & 79,5 & 7.119 & 478.579 & 67,2 \\
\hline Até 5.000 & 449 & 44.116 & 98,3 & 442 & 26.026 & 58,9 & 456 & 27.227 & 59,7 \\
\hline $5.001-10.000$ & 545 & 74.531 & 136,8 & 583 & 43.186 & 74,1 & 699 & 43.491 & 62,2 \\
\hline $10.001-50.000$ & 652 & 263.888 & 404,7 & 1.105 & 147.911 & 133,9 & 1.592 & 146.777 & 92,2 \\
\hline $50.001-100.000$ & 629 & 131.156 & 208,5 & 1.805 & 71.343 & 39,5 & 1.995 & 70.386 & 35,3 \\
\hline 100.000 ou + & 450 & 359.489 & 798,9 & 2.107 & 191.678 & 91,0 & 2.377 & 190.698 & 80,2 \\
\hline Centro-Oeste & 1.188 & 452.743 & 381,1 & 4.050 & 242.009 & 59,8 & 5.849 & 237.242 & 40,6 \\
\hline Até 5.000 & 145 & 18.978 & 130,9 & 287 & 10.622 & 37,0 & 479 & 10.737 & 22,4 \\
\hline $5.001-10.000$ & 110 & 33.137 & 301,2 & 231 & 18.788 & 81,3 & 675 & 18.683 & 27,7 \\
\hline $10.001-50.000$ & 286 & 127.530 & 445,9 & 463 & 70.559 & 152,4 & 1.337 & 70.068 & 52,4 \\
\hline $50.001-100.000$ & 387 & 58.300 & 150,6 & 1.414 & 30.096 & 21,3 & 1.516 & 26.984 & 17,8 \\
\hline $100.000 \mathrm{ou}+$ & 260 & 214.798 & 826,1 & 1.655 & 111.944 & 67,6 & 1.842 & 110.770 & 60,1 \\
\hline
\end{tabular}

(continua)

\section{Desenho da amostra do SB Brasil 2003 e estratégias de cálculo dos pesos amostrais}

O SB Brasil 2003 utilizou uma amostra probabilística com estratificação e conglomeração. A população de referência é constituída pelas pessoas dos seguintes grupos etários: 18 a 36 meses; 5 anos; 12 anos; 15 a 19 anos; 35 a 44 anos; e 65 a 74 anos. A amostra foi estratificada por macrorregião e, em cada uma delas, por porte populacional dos municípios (até 5.000 habitantes; 5.001 a 10.000 habitantes; 10.001 a 50.000 habitantes;
50.001 a 100.000 habitantes; e mais de 100.000 habitantes). Em cada estrato de porte populacional foram, a princípio, selecionados $10 \mathrm{mu}$ nicípios e, em cada município foram escolhidos os seguintes conglomerados: setores censitários, quadras ou vilas, domicílios e estabelecimentos de ensino (escolas e creches).

No entanto, o manual do coordenador 14 , ao descrever a seleção das unidades primárias de amostragem (UPA), indica que as capitais de todas as Unidades da Federação foram incluídas na amostra, não fazendo parte do sorteio de municípios. Portanto, no estrato de maior por- 
Tamanho da amostra, população em 2000 e peso relativo por faixas etárias, segundo as macrorregiões e estratos de porte populacional.

\begin{tabular}{|c|c|c|c|c|c|c|c|c|c|}
\hline \multirow{3}{*}{$\begin{array}{l}\text { Macrorregiões e } \\
\text { estratos de porte } \\
\text { populacional } \\
\text { (habitantes) }\end{array}$} & \multicolumn{9}{|c|}{ Faixa etária } \\
\hline & \multicolumn{3}{|c|}{ 15-19 anos } & \multicolumn{3}{|c|}{$35-44$ anos } & \multicolumn{3}{|c|}{$65-74$ anos } \\
\hline & Amostra & População * & $\begin{array}{c}\text { Peso } \\
\text { relativo ** }\end{array}$ & Amostra & População * & $\begin{array}{c}\text { Peso } \\
\text { relativo ** }\end{array}$ & Amostra & População * & $\begin{array}{c}\text { Peso } \\
\text { relativo ** }\end{array}$ \\
\hline Total & 16.833 & 17.939 .815 & $1.065,8$ & 13.431 & 22.808 .223 & $1.698,2$ & 5.349 & 6.323 .408 & $1.182,2$ \\
\hline Norte & 3.877 & 1.524 .484 & 393,2 & 2.486 & 1.420 .318 & 571,3 & 746 & 303.763 & 407,2 \\
\hline Até 5.000 & 680 & 45.175 & 66,4 & 458 & 41.217 & 90,0 & 122 & 10.916 & 89,5 \\
\hline $5.001-10.000$ & 727 & 97.213 & 133,7 & 430 & 83.794 & 194,9 & 160 & 21.855 & 136,6 \\
\hline $10.001-50.000$ & 852 & 553.086 & 649,2 & 518 & 466.225 & 900,0 & 150 & 111.822 & 745,5 \\
\hline $50.001-100.000$ & 838 & 220.581 & 263,2 & 575 & 191.049 & 332,3 & 166 & 42.286 & 254,7 \\
\hline $100.000 \mathrm{ou}+$ & 780 & 608.429 & 780,0 & 505 & 638.033 & $1.263,4$ & 148 & 116.884 & 789,8 \\
\hline Nordeste & 3.998 & 5.571 .708 & $1.393,6$ & 3.370 & 5.513 .073 & $1.635,9$ & 1.446 & 1.667 .384 & $1.153,1$ \\
\hline Até 5.000 & 711 & 128.633 & 180,9 & 548 & 114.200 & 208,4 & 249 & 43.366 & 174,2 \\
\hline $5.001-10.000$ & 776 & 359.952 & 463,9 & 551 & 310.459 & 563,4 & 253 & 117.759 & 465,5 \\
\hline $10.001-50.000$ & 749 & 2.416 .235 & $3.225,9$ & 618 & 2.095 .534 & $3.390,8$ & 259 & 763.970 & $2.949,7$ \\
\hline $50.001-100.000$ & 969 & 742.010 & 765,7 & 881 & 722.961 & 820,6 & 416 & 217.288 & 522,3 \\
\hline $100.000 \mathrm{ou}+$ & 793 & 1.924 .878 & $2.427,3$ & 772 & 2.269 .919 & $2.940,3$ & 269 & 525.001 & $1.951,7$ \\
\hline Sudeste & 2.981 & 7.155 .091 & $2.400,2$ & 2.340 & 10.580 .065 & $4.521,4$ & 1.052 & 2.994 .665 & $2.846,6$ \\
\hline Até 5.000 & 677 & 162.113 & 239,5 & 487 & 209.345 & 429,9 & 205 & 78.096 & 381,0 \\
\hline $5.001-10.000$ & 683 & 307.811 & 450,7 & 513 & 388.064 & 756,5 & 238 & 138.388 & 581,5 \\
\hline $10.001-50.000$ & 679 & 1.359 .052 & $2.001,5$ & 534 & 1.821 .075 & $3.410,3$ & 286 & 574.498 & $2.008,7$ \\
\hline $50.001-100.000$ & 391 & 736.638 & $1.884,0$ & 366 & 1.061 .078 & $2.899,1$ & 158 & 288.090 & $1.823,4$ \\
\hline $100.000 \mathrm{ou}+$ & 551 & 4.589 .477 & $8.329,4$ & 440 & 7.100 .503 & $16.137,5$ & 165 & 1.915 .593 & $11.609,7$ \\
\hline Sul & 3.841 & 2.451 .895 & 638,3 & 3.189 & 3.702 .974 & $1.161,2$ & 1.374 & 1.025 .853 & 746,6 \\
\hline Até 5.000 & 704 & 131.064 & 186,2 & 516 & 196.436 & 380,7 & 231 & 69.386 & 300,4 \\
\hline $5.001-10.000$ & 665 & 212.672 & 319,8 & 407 & 307.423 & 755,3 & 184 & 103.677 & 563,5 \\
\hline $10.001-50.000$ & 751 & 728.887 & 970,6 & 633 & 1.060 .592 & $1.675,5$ & 290 & 311.578 & $1.074,4$ \\
\hline $50.001-100.000$ & 896 & 363.703 & 405,9 & 769 & 553.576 & 719,9 & 291 & 144.581 & 496,8 \\
\hline $100.000 \mathrm{ou}+$ & 825 & 1.015 .569 & $1.231,0$ & 864 & 1.584 .947 & $1.834,4$ & 378 & 396.631 & $1.049,3$ \\
\hline Centro-Oeste & 2.136 & 1.236 .637 & 578,9 & 2.046 & 1.591 .793 & 778,0 & 731 & 331.743 & 453,8 \\
\hline Até 5.000 & 298 & 52.154 & 175,0 & 278 & 68.127 & 245,1 & 103 & 18.432 & 179,0 \\
\hline $5.001-10.000$ & 273 & 88.919 & 325,7 & 269 & 114.003 & 423,8 & 95 & 28.674 & 301,8 \\
\hline $10.001-50.000$ & 506 & 343.407 & 678,7 & 555 & 439.176 & 791,3 & 195 & 105.315 & 540,1 \\
\hline $50.001-100.000$ & 512 & 135.616 & 264,9 & 467 & 166.813 & 357,2 & 172 & 32.623 & 189,7 \\
\hline $100.000 \mathrm{ou}+$ & 547 & 616.541 & $1.127,1$ & 477 & 803.674 & $1.684,9$ & 166 & 146.699 & 883,7 \\
\hline
\end{tabular}

* População em 2000 (fonte: Censo Demográfico de 2000);

** Razão entre o número de pessoas na população em 1ㅇ de setembro de 2000 e na amostra efetiva do SB Brasil 2003.

te populacional (mais de 100 mil habitantes) de cada macrorregião, os municípios de capital não correspondem à UPA e sim a estratos de seleção, uma vez que foram incluídos na amostra, sem procedimento de seleção aleatória. Além disso, no estrato de maior porte populacional da macrorregião Nordeste, as nove capitais foram incluídas, tendo sido selecionado apenas um município, o que viola um princípio básico de amostragem: o tamanho da amostra deve ser maior que um, para permitir o cálculo da variabilidade. Este problema pode ser corrigido pelo agrupamento dos municípios deste estrato (exceto os de capital) com os do estrato de porte populacional imediatamente inferior (50.001 a 100.000 habitantes). Dessa forma, são 51 os estratos da amostra, correspondentes a: 27 capitais; mais 20 estratos correspondentes à combinação das regiões Norte, Sudeste, Sul e Centro-Oeste com 5 estratos de porte populacionais; mais quatro estratos de porte populacional na Região Nordeste.

Selecionados ou incluídos os municípios, os demais estágios de seleção foram diferenciados em dois grupos de estratos: (1) os de até $50 \mathrm{mil}$ habitantes; e (2) os de mais de 50 mil habitantes. Dentro dos municípios com até 50 mil ha- 
Variação, média e coeficiente de variação do peso relativo, segundo os grupos etários.

\begin{tabular}{|c|c|c|c|}
\hline Grupos etários & Variação do peso relativo & Média & Coeficiente de variação (CV) \\
\hline 18-36 meses & 63,1 e $3.733,5$ & 534,3 & $3.187 \%$ \\
\hline 5 anos & 21,3 e 744,5 & 129,3 & $3.413 \%$ \\
\hline 12 anos & 17,8 a 363,6 & 102,0 & $3.676 \%$ \\
\hline 15-19 anos & 66,4 a $8.329,4$ & $1.065,8$ & $3.865 \%$ \\
\hline $35-44$ anos & 90,0 a $16.137,5$ & $1.698,2$ & $3.962 \%$ \\
\hline 65- 74 anos & 89,5 a $11.609,7$ & $1.182,2$ & $2.520 \%$ \\
\hline
\end{tabular}

bitantes, foram selecionadas quadras e escolas (segundo estágio). Dentro das quadras, foram selecionados os domicílios (terceiro estágio) em que foram entrevistados os indivíduos. E, dentro das escolas, foram selecionados, no terceiro estágio, os alunos com a idade de interesse (12 anos). Para os municípios com mais de 50 mil habitantes foram selecionados setores, escolas e creches (primeiro estágio, nos municípios de capital, e segundo estágio nos demais). Dentro dos setores, foram selecionadas quadras (segundo ou terceiro estágio) e posteriormente domicílios (terceiro ou quarto estágio) em que foram entrevistados os indivíduos. E, dentro das escolas e creches, foram selecionados os alunos com as idades de interesse (5 e 12 anos). A Tabela 3 fornece indicação dos estratos, das unidades elementares da amostra e dos distintos estágios de seleção para os diferentes grupos definidos no plano de amostragem.

Para o cálculo dos pesos amostrais, definidos como o inverso das probabilidades de inclusão das unidades elementares na amostra, é necessário identificar as unidades de seleção de cada estágio da amostra, a saber: municípios, escolas, creches, setores, quadras/vilas, domicílios e alunos. Tais unidades, por sua vez, variam de acordo com o porte do município, com o fato de o município ser de capital ou não, e com o fato de o indivíduo ter sido entrevistado em domicílio ou estabelecimento de ensino, como indica a Tabela 3.

Partindo do pressuposto de que a coleta de dados foi feita exatamente como o manual do coordenador 14 estabelece, é possível obter as informações necessárias para cálculo dos pesos amostrais. De fato, o referido manual previu um modelo de relatório de campo no qual deveriam constar informações que possibilitariam a recuperação dos dados necessários ao cálculo das probabilidades de inclusão de quadras urbanas e vilas rurais sorteadas, além do número total de domicílios identificados, e do período de coleta de dados nos domicílios e nas escolas, indicando que esses relatórios seriam a fonte preferencial para obtenção dos dados requeridos. Porém, na ausência desses dados nos relatórios ou no caso dos relatórios não serem recuperados, a opção que resta é buscar informações em fontes exógenas (IBGE e MEC) e pressupor que as regras do referido manual foram estritamente seguidas. Além disso, como não foi prevista a inclusão de informações sobre as escolas selecionadas nem sobre os respectivos números de alunos nos relatórios ou na folha do questionário de coleta de dados, só restam para esses casos os dados do Censo Escolar do MEC.

Para municípios, as informações necessárias para determinar as probabilidades de inclusão constam do manual do coordenador 14 . No caso dos municípios de capital, a probabilidade de inclusão é igual a um, pois eles foram incluídos na amostra sem seleção. Para os municípios selecionados por processo aleatório, a probabilidade de inclusão é proporcional à estimativa populacional dos municípios para 1o de julho de 1999 . As referidas probabilidades de inclusão constam das expressões 1 e 2 da Figura 1.

No caso da seleção de setores, aplicável apenas a municípios com mais de 50 mil habitantes, embora não conste do manual do coordenador, foi feita uma seleção equiprovável e a probabilidade de inclusão consta da expressão 3 da Figura 1. Os dados da Base Operacional Geográfica (BOG) do IBGE são suficientes para determinar as probabilidades de cada setor selecionado. Os códigos desses setores constam, exceto por alguns erros de digitação, da base de dados do SB Brasil 2003. A expectativa é que o relatório da equipe técnica responsável pela seleção da amostra permita corrigir esses poucos erros de digitação.

A seleção de quadras/vilas foi feita com reposição e eqüiprobabilidade, e as probabilidades de inclusão constam das expressões 4 e 5 da Figura 1. Há pequena variação na forma de 
Estratos, unidades elementares e estágios de seleção por porte do município, condição do município (capital ou não) e local da entrevista.

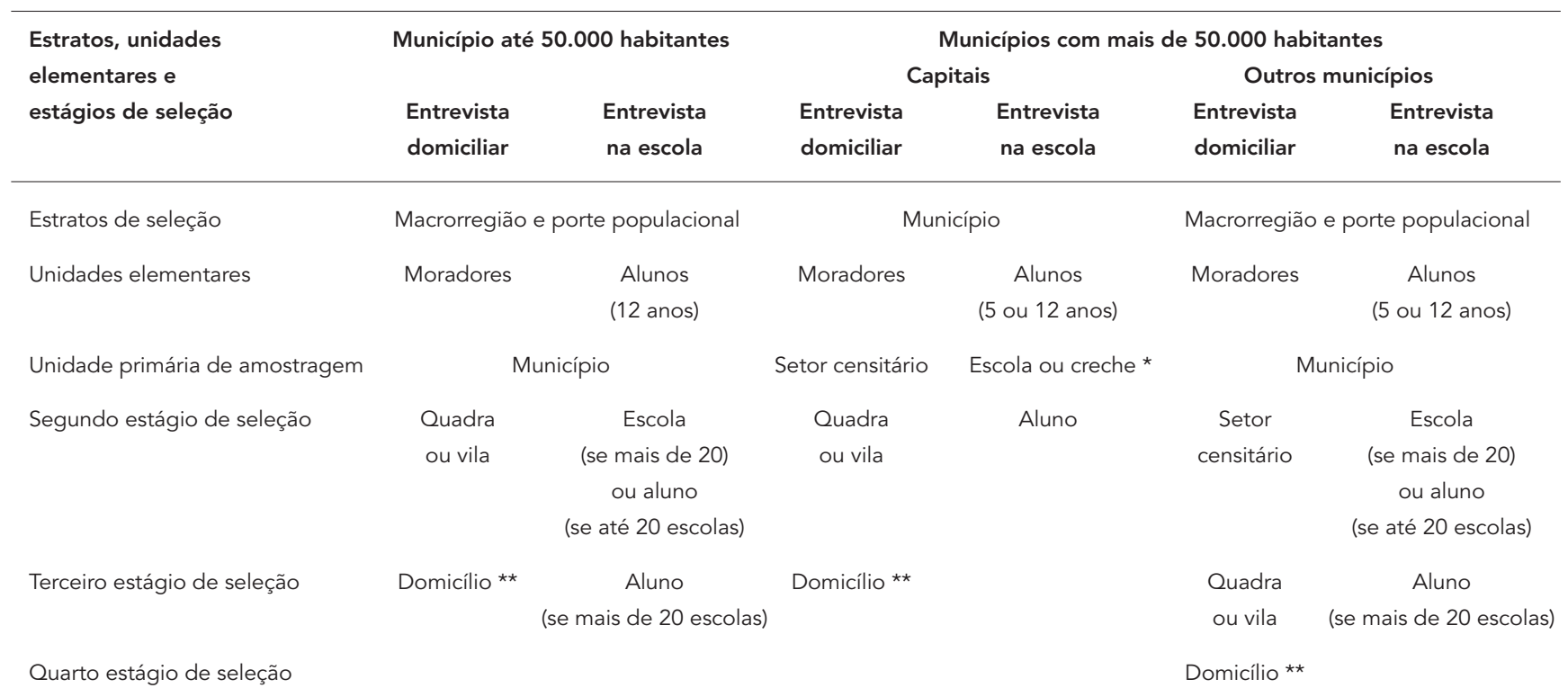

* Supõe-se que existam mais de 20 escolas e mais de 20 creches em todas as capitais, caso contrário valem as regras para outros municípios com mais de 50.000 habitantes. Este é, em princípio, o único caso em que será necessário usar simulação estatística para avaliar o efeito de diferentes agrupamentos de alunos por escola ou creche;

** De fato, os domicílios não são sempre selecionados. Para alguns grupos etários, todos os moradores de todos os domicílios são entrevistados (os que se recusarem são considerados como não-resposta). No entanto, para certos grupos etários mais freqüentes na população foi estabelecida uma fração de amostragem, que implica a seleção de um a cada dois domicílios. Neste caso, há seleção de domicílios e são entrevistados todos os moradores do grupo etário, desde que dêem seu consentimento.

calcular numerador e denominador em função da maneira como é calculada a média de domicílios por quadra. Nos municípios até 50 mil habitantes, onde não há seleção de setores, essa média parte do pressuposto de que o tamanho médio do domicílio é igual a quatro pessoas (expressão 5.1 da Figura 1). Nos demais municípios, onde os setores foram selecionados, essa média parte do pressuposto de que os setores têm, em média, 300 domicílios (expressão 5.3 da Figura 1).

Há, também, uma certa imprecisão na definição do tamanho da amostra de domicílios (usados nas expressões 5.2 e 5.4 da Figura 1) no manual do coordenador 14, em função de ajustes feitos (correção de população finita, população média dos municípios em cada estrato etc.). Essa imprecisão fica evidente nos dados apresentados na Tabela 4. Mas isso não é importante para calcular as probabilidades de inclusão, pois será usado o tamanho efetivo da amostra, determinado com base do banco de dados do SB Brasil 2003. O uso do tamanho efetivo da amostra corresponde a uma correção de não-resposta, visto que irá alterar o peso amostral para com- pensar a diferença entre a amostra desejada e a efetiva (que, no caso, é a não-resposta).

Voltando à estratégia para calcular as probabilidades de inclusão, serão usados os dados dos relatórios das equipes de campo. Caso eles não existam, o número de quadras/vilas pode ser obtido nos mapas dos setores selecionados (municípios com mais de 50.000 habitantes) ou nos Mapas Municipais para fins Estatísticos (MME), disponíveis na BOG do IBGE.

Para a seleção dos domicílios, deve-se considerar duas possibilidades que dependem do estrato da amostra e da faixa etária em consideração, como indica a expressão 6 da Figura 1: (1) não há seleção de domicílios e todas as unidades domiciliares existentes na quadra entram na amostra (probabilidade de inclusão igual a um), ou (2) a seleção é realizada de forma sistemática, sendo pesquisado um a cada dois domicílios (probabilidade de inclusão igual a 1/2). As frações de amostragem de $100 \%$ ou $50 \%$ estão indicadas na Tabela 4. Também nesse estágio de seleção não é necessário contar os domicílios das quadras e vilas selecionados, bastando contá-los na base de dados do SB Brasil 2003 e aplicar a fração 
Figura 1

Esquema probabilístico da amostra do SB Brasil 2003.

\section{A. Probabilidades de inclusão na amostra para pessoas entrevistadas nos domicílios}

Probabilidade de inclusão de municípios

(1) $\quad P$ (município de capital $=1$ )

(2) $P$ (outros municípios) $=\frac{n \times \text { população do município em 01/07/99 }}{\text { população do estrato em 01/07/99 }}$, onde

n é o número de municípios selecionados no estrato e, de um modo geral igual a 10, exceto para o maior estrato de porte populacional das macrorregiões Norte, Sudeste, Sul e Centro-Oeste, em que n é igual a 3, 6, 7 e 6, respectivamente, bem como para o quarto estrato de porte populacional (50.001 a 100.000 habitantes) do Nordeste onde n é igual 11 (pela inclusão do único município de mais de 100 mil habitantes selecionado).

Probabilidade de inclusão de setores (apenas para municípios com mais de $\mathbf{5 0 . 0 0 0}$ habitantes):

(3) $P($ setor $)=\frac{10}{\text { número de setores do município }}$, se seleção com eqüiprobabilidade; ou

\section{Probabilidade de inclusão de quadras ou vilas rurais:}

(4) $\mathrm{P}$ (quadras/vila) $=1$, se o município tiver até 10 quadras; ou

(5) $P$ (quadras/vila) $=\frac{T A_{q v}}{T P_{q v}}$, onde

$\mathrm{TA}_{\mathrm{qv}}$ é o tamanho da amostra de quadras e vilas; e

$\mathrm{TP}_{\mathrm{qv}}$ é o tamanho da população de quadras e vilas.

Para o cálculo de $\mathrm{TA}_{\mathrm{qv}}$ e $\mathrm{TP}_{\mathrm{qv}}$ as regras mudam de acordo com a população municipal.

Para municípios com até 50 mil habitantes, não há seleção de setores e o tamanho da amostra depende do número médio de domicílios (MDQ) por quadra/vila, como indicado nas expressões 5.1 e 5.2 :

(5.1) $\mathrm{MDQ}=\frac{\frac{\text { População total do municipio }}{4}}{\text { Número de quadras/vilas do município }}$

(5.2) $\mathrm{TA}_{\mathrm{qv}}=\frac{\mathrm{TA}_{\mathrm{d}}}{\mathrm{MDQ}}$ onde $\mathrm{TA}_{\mathrm{d}}$ é o tamanho da amostra de domicílios indicado na Tabela 4.

Para municípios com mais de 50 mil habitantes, há seleção de setores e o tamanho da amostra depende do número médio de domicílios (MDQ) por quadra/vila nos 10 setores selecionados, como indicado nas expressões 5.3 e 5.4 :

(5.3) $\mathrm{MDQ}=\frac{300 \times 10}{\text { Número de quadras/vilas nos } 10 \text { setores selecionados }}$
(5.4) $\mathrm{TA}_{\mathrm{qv}}=\frac{\mathrm{TA}_{\mathrm{d}}}{\mathrm{MDQ}}$ onde $\mathrm{TA}_{\mathrm{d}}$ é o tamanho da amostra de domicílios indicado na Tabela 4.

\section{Probabilidade de inclusão dos domicílios}

(6) $\mathrm{P}($ domicílio $)=1$ ou $1 \frac{1}{2}$, de acordo com as frações de amostragem indicadas na Tabela 4.

\section{Probabilidade de inclusão das unidades elementares (moradores)}

Como os moradores não são alvo de seleção, pois todos os residentes em domicílios selecionados que aceitaram a pesquisa foram examinados, sua probabilidade de inclusão corresponde ao produto das probabilidades de inclusão nos diferentes estágios de seleção.

\section{B. Probabilidades de inclusão para crianças selecionadas em estabelecimentos de ensino}

Probabilidade de inclusão de municípios

São as mesmas definidas anteriormente nas expressões (1) e (2).

(continua) 
Probabilidade de inclusão de escolas (para seleção de crianças de 12 anos)

(7) $\mathrm{P}($ escola $)=1$, se o município tiver até 20 escolas de 1 o grau freqüentadas por crianças de 12 anos; ou

(8) $\quad \mathrm{P}($ escola $)=\frac{20 \times \text { número de alunos da escola }}{\text { Total de alunos de } 12 \text { nas escolas do município }}$

Probabilidade de inclusão de creches (crianças de 5 anos e municípios com população $>50.000$ )

(9) $P($ creche $)=1$, se o município tiver até 20 creches freqüentadas por crianças de 5 anos; ou

(10) $P($ creche $)=\frac{\text { Tamanho da amostra de alunos }}{\text { Total de alunos de } 5 \text { anos nas creches do município }}$

Probabilidade de inclusão de crianças de 5 e 12 anos

(11) $P($ criança $)=\frac{\text { Tamanho de amostra de alunos }}{\text { Total de alunos nas escolas selecionadas (ou incluídas) }}$

Probabilidade de inclusão das unidades elementares (alunos)

A probabilidade de inclusão dos alunos corresponde ao produto das probabilidades de inclusão do município, da escola (ou creche) e da criança.

de amostragem correspondente à idade das pessoas entrevistadas.

As pessoas de 12 anos foram sempre selecionadas em escolas. As crianças de 5 anos foram selecionadas nos domicílios, se o município tinha até 50.000 habitantes, ou em creches no caso de municípios com mais de 50.000 habitantes. Para os estabelecimentos de ensino (escolas e creches), as probabilidades de inclusão são dadas pelas expressões 7 a 10 da Figura 1. As expressões 7 e 9 aplicam-se aos municípios com até 20 escolas ou creches, respectivamente, caso no qual os estabelecimentos de ensino são incluídos na amostra com probabilidade igual a um. As expressões 8 e 10 da Figura 1 indicam que, no caso de mais de 20 escolas e mais de 20 creches, elas foram selecionadas com probabilidade proporcional ao seu número de alunos. Para determinar as probabilidades de inclusão das expressões $8 \mathrm{e}$ 10 serão preferencialmente usadas as informações dos relatórios das equipes de campo. $\mathrm{Na}$ ausência dessas informações, a melhor aproximação possível é usar o número de alunos de 6a série de cada escola (ou de cinco anos na pré-escola) selecionada e o número de alunos de 6 a série de todas as escolas (ou de 5 anos de todas as pré-escolas) do município, disponibilizados pelo Censo Escolar de 2003 (MEC).

Para crianças de 5 e 12 anos selecionadas em estabelecimentos de ensino, foi adotado um procedimento de seleção sistemática com eqüiprobabilidade, independentemente do número de estabelecimentos de ensino do município. A probabilidade de inclusão de um aluno consta da expressão 11 da Figura 1. Também, neste caso, será usado o tamanho da amostra efetiva como numerador (para correção das não-respostas) e o denominador, obtido da mesma fonte usada para o numerador das expressões 8 e 10 da Figura 1, corresponde à soma de alunos de 12 anos (ou 5 anos) das escolas (ou creches) selecionadas ou incluídas na amostra.

Enfim, a fonte de informações preferencial para o cálculo das probabilidades de inclusão são os relatórios das equipes de campo. Todavia, não sendo possível recuperar todas as informações necessárias nesses relatórios, o uso de informações do IBGE e do INEP permitirá obter probabilidades de inclusão aproximadas em cada estágio de seleção. Com essas probabilidades de inclusão, exatas ou aproximadas, o peso amostral de cada unidade elementar da amostra pode ser obtido pelo inverso do produto das suas probabilidades de inclusão em cada estágio da amostra, como indicado na Figura 1.

Após o cálculo exato ou aproximado dos pesos amostrais, um processo de calibração desses pesos deve ser aplicado para assegurar que as estimativas reproduzam os totais populacionais por sexo e faixa de idade, conhecidos para todos os estratos de seleção mediante a projeção da população feita pelo IBGE, para o ano de 2003.

Entretanto, calcular os pesos amostrais não é suficiente. É preciso identificar cada estrato de 
Número previsto de domicílios na amostra, tamanho da amostra e fração de amostragem por faixa etária, segundo as macrorregiões e os estratos de porte populacional.

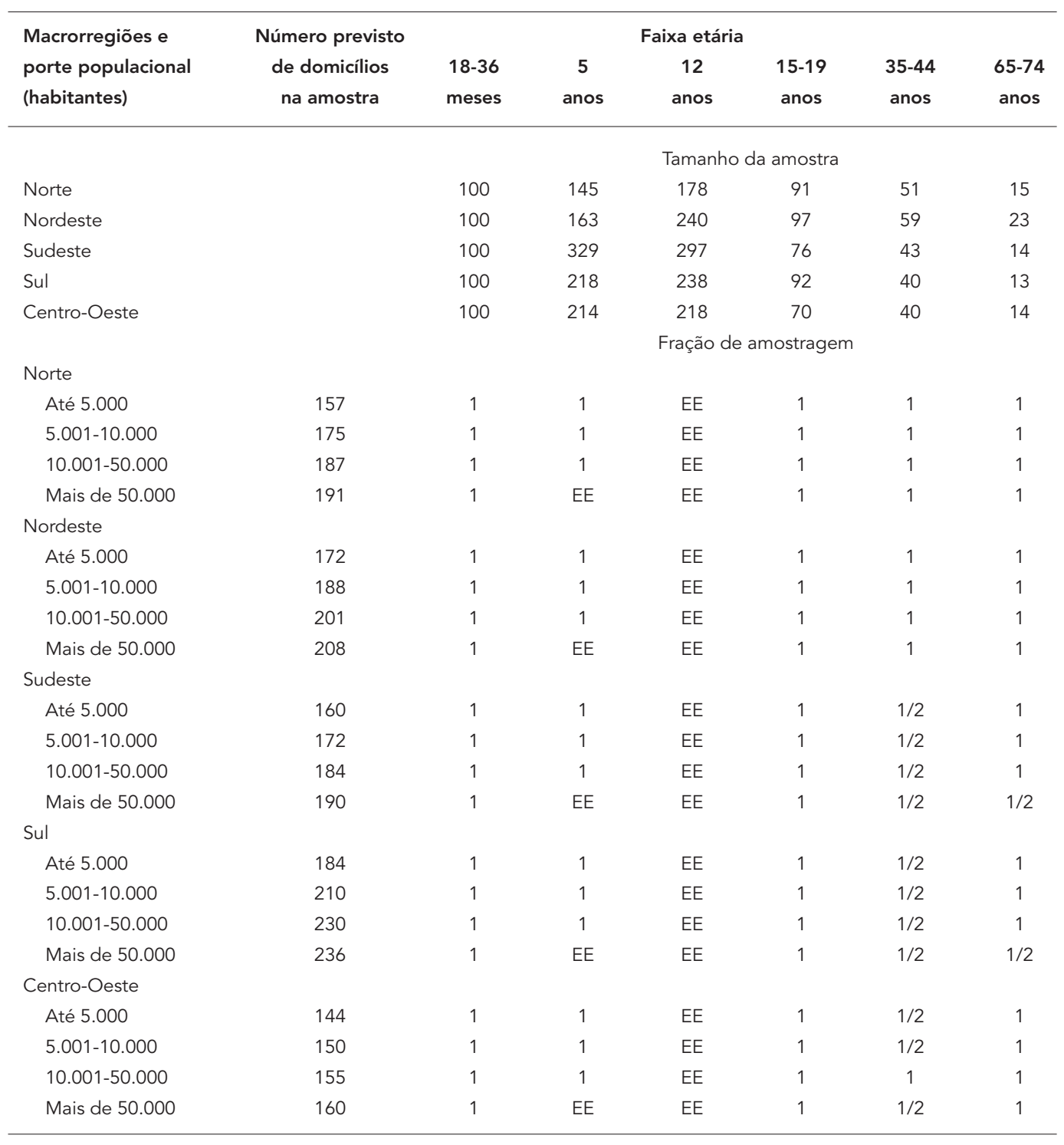

EE: pesquisado em estabelecimento de ensino.

Adaptado das Tabelas 2, 4 e 5 do manual do coordenador 14

seleção e todas as suas unidades primárias de amostragem, a fim de viabilizar o cálculo de variâncias e demais estatísticas delas dependentes.

O manual do coordenador 14 esclarece que a amostra tem 25 estratos (5 macrorregiões x 5 estratos de porte populacional). Já o texto anterior mostra que, na realidade, a amostra do SB Brasil 2003 foi selecionada de uma população estratificada em 51 estratos ( $=27$ capitais +20 estratos de porte populacional nas macrorregiões Norte, Sudeste, Sul e Centro-Oeste +4 estratos de porte populacional na macrorregião Nordeste, onde o estrato de maior porte populacional foi agrupado ao estrato anterior, como indicado anteriormente).

Também não são 250 UPA (10 municípios x 25 estratos), como está descrito no referido manual, apesar de o número exato de UPA ainda não ter sido determinado. A Tabela 3 mostra que, de acordo com o estrato de seleção, a UPA pode ser o município, o setor censitário, a escola ou a creche. Como o número de escolas e creches ain- 
da não foi determinado, o número total de UPA ainda é desconhecido. O problema, nesse caso, é que o arquivo de dados não identifica cada estabelecimento de ensino (escola ou creche) selecionado, apenas identificando o setor, a quadra ou vila e o tipo de estabelecimento de ensino. Os relatórios das equipes de campo constituem-se na fonte ideal para determinar quais estabelecimentos de ensino foram selecionados em cada município da amostra. Na sua ausência, a única alternativa é fazer uso da técnica de simulação estatística para identificar o impacto de diferentes agrupamentos (aleatoriamente construídos em cada município) de alunos por escola (ou creche) sobre as variâncias das principais variáveis da pesquisa. Se o impacto não for grande, pode-se determinar - e identificar no arquivo os agrupamentos a serem utilizados no processo de estimação de variâncias e estatísticas delas derivadas.

\section{Resumo}

$A$ Pesquisa sobre as Condições de Saúde Bucal da População Brasileira (SB Brasil 2003) foi a mais abrangente pesquisa sobre as condições de saúde bucal realizada no Brasil até hoje. Métodos de amostragem probabilística foram utilizados para assegurar que os dados coletados representariam a população das faixas etárias escolhidas nas cinco regiões do Brasil. No entanto, isso não foi possível, pois o processo de amostragem da pesquisa não foi finalizado. Sua conclusão pressupõe o cálculo dos pesos amostrais e a identificação das demais variáveis estruturais da amostra (estratos de seleção e unidades primárias de amostragem). Este artigo descreve o desenho de amostra desta pesquisa, formula as probabilidades de inclusão nos diversos estágios de seleção e propõe estratégias de cálculo dos pesos amostrais. A solução para determinar os pesos amostrais e identificar as demais variáveis estruturais da amostra, proposta no artigo, parte da recuperação de informações que deveriam ter sido registradas nos relatórios produzidos durante a pesquisa de campo e, em sua ausência, em informações disponíveis no Instituto Brasileiro de Geografia e Estatística e no Ministério da Educação, como aproximações válidas.

Saúde Bucal; Amostragem; Nível de Saúde; População

\section{Conclusão}

Trata-se, portanto, de uma proposta de correção trabalhosa, que requer a busca de informações junto às coordenações municipais, estaduais e regionais do SB Brasil 2003 e, para completar as informações faltantes, o processamento de bases de dados exógenas ao projeto.

Contudo, o esforço de completar o processo de amostragem probabilística do SB Brasil 2003, calculando pesos amostrais e identificando as demais informações estruturais de sua amostra, justifica-se para ampliar o uso das informações coletadas, que, em última instância, é o que justifica o custo de qualquer pesquisa.

Além disso, inferências válidas para a população, então tecnicamente viáveis, permitirão que pesquisadores e gestores possam analisar as reais condições de saúde bucal no país e traçar metas para seu melhoramento.

\section{Colaboradores}

M. C. Portela e R. C. S. Queiroz identificaram a falta de pesos amostrais no arquivo de dados do SB Brasil 2003. M. T. L. Vasconcellos orientou o estudo dos documentos existentes sobre o desenho da amostra e identificou os demais problemas do desenho. Os três autores planejaram os procedimentos de correção dos problemas identificados. R. C. S. Queiroz escreveu a primeira versão do manuscrito, que foi revisado e aprovado pelos demais autores.

\section{Agradecimentos}

Os autores agradecem à Maria Cecília Goi Porto Alves as informações relativas ao teste piloto realizado em Diadema (São Paulo) e Canela (Rio Grande do Sul), e a Angelo Giuseppe Roncalli da Costa Oliveira os esclarecimentos prestados sobre a técnica de seleção dos setores, além de sua cooperação ao fornecer a listagem dos setores selecionados.

Este trabalho recebeu apoio da Fundação Carlos Chagas Filho de Amparo à Pesquisa do Estado do Rio de Janeiro (FAPERJ; processo E-26/171.336/2006), e os seus autores são bolsistas do Conselho Nacional de Desenvolvimento Científico e Tecnológico (CNPq). 


\section{Referências}

1. Divisão Nacional de Saúde Bucal, Ministério da Saúde. Levantamento epidemiológico em saúde bucal: Brasil, zona urbana. Brasília: Ministério da Saúde; 1988.

2. Oliveira AGR, Unfer B, Costa ICC, Arcieri RM, Guimaraes LOC, Saliba NA. Levantamentos epidemiológicos em saúde bucal: análise da metodologia proposta pela Organização Mundial da Saúde. Rev Bras Epidemiol 1998; 1:177-89.

3. Fédèration Dentaire Internationale. Global goals for oral health in the year 2000. Int Dent J 1982; 32:74-7.

4. Costa JFR, Chagas LD, Silvestre RM, organizadores. A política nacional de saúde bucal do Brasil: registro de uma conquista histórica. Brasília: Organização Pan-Americana da Saúde/Ministério da Saúde; 2006. (Serie Técnica Desenvolvimento de Sistemas e Serviços de Saúde, 11).

5. Coordenação de Saúde Bucal, Departamento de Assistência e Promoção à Saúde, Secretaria de Assistência à Saúde, Ministério da Saúde. Levantamento epidemiológico em saúde bucal: 1a etapa - cárie dental. Brasília: Ministério da Saúde; 1996.

6. Departamento de Atenção Básica, Secretaria de Atenção à Saúde, Ministério da Saúde. Projeto SB Brasil 2003. Condições de saúde bucal da população brasileira, 2002-2003: resultados principais. Brasília: Ministério da Saúde; 2004.
7. Antunes JLF, Peres MA, Mello TRC, Waldman EA Multilevel assessment of determinants of dental caries experience in Brazil. Community Dent Oral Epidemiol 2006; 34:146-52.

8. Antunes JLF, Peres MA, Mello TRC. Determinantes individuais e contextuais da necessidade de tratamento odontológico na dentição decídua no Brasil. Ciênc Saúde Coletiva 2006; 11:79-87.

9. Matos DL, Lima-Costa MF. Auto-avaliação da saúde bucal entre adultos e idosos residentes na Região Sudeste: resultados do Projeto SB-Brasil, 2003. Cad Saúde Pública 2006; 22:1699-707.

10. Narvai P, Frazão P, Roncalli A, Antunes J. Cárie dentária no Brasil: declínio, polarização, iniqüidade e exclusão social. Rev Panam Salud Pública 2006; 19:385-93

11. Kiar AN. Observations et expériences concernant les dénombrements représentatifs. Bulletin of the International Statistical Institute 1895; 9:176-205.

12. Cochran WG. Sampling techniques. 3rd Ed. New York: John Wiley and Sons; 1977.

13. Pessoa DGC, Silva PLN. Análise de dados amostrais complexos. São Paulo: Associação Brasileira de Estatística; 1998.

14. Área Técnica de Saúde Bucal, Departamento de Atenção Básica, Secretaria Políticas de Saúde, Ministério da Saúde. Projeto SB2000. Condições de saúde bucal da população brasileira no ano 2000 manual do coordenador. Brasília: Ministério da Saúde; 2001.

Recebido em 02/Jan/2008

Versão final reapresentada em 15/Mai/2008

Aprovado em 06/Jun/2008 